\title{
Clinical Experience in Patients with Ocular Burns Treated with Boston Type I Keratoprosthesis Implantation with or Without Prophylactic Ahmed Glaucoma Valve Implantation
}

\author{
Jianjun $\mathrm{Gu} \cdot$ Yuying Zhang $\cdot$ Jiajie Zhai $\cdot$ Lixia Lin $\cdot$ Zhancong Ou $\cdot$ \\ Ting Huang $\cdot$ Miao Chen $\cdot$ Jin Zhou $\cdot$ Liangbo Zeng $\cdot$ Yuwei Xu \\ Jiaqi Chen (D)
}

Received: October 30, 2021 / Accepted: December 6, 2021 / Published online: December 23, 2021

(C) The Author(s) 2021

\begin{abstract}
Introduction: To compare outcomes in eyes with ocular burns following Boston Type I keratoprosthesis (KPro) implantation with and without prophylactic pars plana tube surgery.

Methods: A retrospective review of patients with ocular burns who underwent KPro surgery at Zhongshan Ophthalmic Center was performed. Twenty-six eyes of 26 patients without a preoperative diagnosis of glaucoma before KPro surgery met the inclusion criteria. Preoperative glaucoma was defined as a history of a durable elevated intraocular pressure (IOP) $\geq 25 \mathrm{mmHg}$ at different time points, which resulted in the introduction of anti-
\end{abstract}

Jianjun Gu, Yuying Zhang, Jiajie Zhai, Lixia Lin and Zhancong Ou contributed equally to this work and all are considered principal authors.

J. Gu · Y. Zhang · L. Lin · Z. Ou · T. Huang ·

M. Chen · J. Zhou $\cdot$ L. Zeng $\cdot$ Y. Xu · J. Chen ( $ه)$

State Key Laboratory of Ophthalmology, Zhongshan

Ophthalmic Center, Sun Yat-Sen University,

Guangzhou 510060, China

e-mail: docjiaqi@163.com

Y. Zhang

Department of Ophthalmology, Guangdong

Women and Children Hospital, Guangzhou 510060,

China

J. Zhai · J. Chen

Guangdong Visual and Ophthalmology Research

Institute, Guangzhou 510060, China glaucoma medication or surgical intervention. Sixteen eyes underwent KPro alone (Group 1), and 10 eyes received KPro with prophylactic pars plana tube surgery (Group 2).

Results: Group 1 and Group 2 were similar in the proportions of the ocular burn type and preoperative clock hours of peripheral anterior synechiae by ultrasound biomicroscopy $(1.88 \pm 1.63$ vs. $2.30 \pm 1.83 ; P=0.54)$. Before KPro surgery, $62.5 \%$ of eyes in Group 1 and $50.0 \%$ of eyes in Group 2 had intraocular surgeries $(P=0.53)$. The follow-up time was 18 months. At the final follow-up time, the two groups had similar visual acuity $(1.34 \pm 0.87$ $\log$ MAR, $1.03 \pm 0.71 \log$ MAR; $P=0.35)$ and eyes with a $C / D$ ratio $\geq 0.8 \quad(7 / 16,2 / 10$; $P=0.21$ ), but more eyes in Group 1 developed glaucoma de novo than eyes in Group 2 (62.5\%, $20 \% ; P=0.04)$ and had undergone secondary glaucoma surgery after KPro implantation (7/16 vs. $0 / 10 ; P=0.02$ ).

Conclusion: In eyes injured with ocular burns, KPro implantation with prophylactic pars plana tube surgery may be a feasible option to rehabilitate visual acuity and decrease the incidence of glaucoma de novo.

Keywords: Boston type I keratoprosthesis; Glaucoma; Ocular burn; Pars plana 


\section{Key Summary Points}

\section{Why carry out this study?}

Boston keratoprosthesis type 1 (KPro) for ocular burns has been reported to be a viable treatment option. However, KPro carries continued risks of glaucoma, compromising visual rehabilitation after an otherwise successful KPro procedure. Management of glaucoma in such eyes is critical yet challenging. Given a high rate of reported de novo glaucoma and fastest glaucoma progression occurred in the burn category, the study compares outcomes in eyes with ocular burns following KPro implantation with and without prophylactic pars plana tube surgery.

\section{What was learned from the study?}

In eyes injured with ocular burns, KPro implantation with prophylactic pars plana tube surgery may be a feasible option to rehabilitate visual acuity and decrease the incidence of de novo glaucoma.

Twenty-six eyes of 26 patients without a preoperative diagnosis of glaucoma before KPro surgery met the inclusion criteria. Preoperative glaucoma was defined as a history of a durable elevated intraocular pressure $(\mathrm{IOP}) \geq 25 \mathrm{mmHg}$ at different time points, which resulted in the introduction of anti-glaucoma medication or surgical intervention. At the 18-month follow-up, a $C / D$ ratio progression of $\geq 0.2$ was observed in $62.5 \%(10 / 16)$ of Group KPro alone and 20.0\% (2/10) of Group KPro combined tube surgery $(P=0.04)$, and more eyes in Group KPro alone had postoperative glaucoma surgery than in Group KPro combined tube surgery $(7 / 16$ vs. $0 / 10 ; P=0.02)$.

\section{INTRODUCTION}

Boston keratoprosthesis type 1 (KPro) for ocular burns has been reported to be a viable treatment option [1], with most patients showing improved postoperative vision. One study reported that patients with ocular burns had relatively good visual results compared with other diagnoses for KPro implantation [2]. Good outcomes were also reported with 100\% retention of the KPro, as the primary penetrating corneal procedure, in patients whose indication was chemical injury [3]. The advantage of a KPro over penetrating keratoplasty includes elimination of corneal graft rejection, decreased dependence on limbal stem cell function, and less concern about systemic immunosuppression. However, sustained intraocular pressure elevation after KPro places visual function in jeopardy because of the potential for optic nerve injury. The KPro patient must adhere indefinitely to lifetime glaucoma evaluation and management. Efforts to stabilize the intraocular pressure (IOP) of patients with KPro implantation should be undertaken as much as possible.

Many corneal disorders in ocular burns for which KPro is indicated are associated with anterior segment abnormalities, such as peripheral anterior synechiae (PAS), a distorted pupil, posterior synechiae and forwards rotation of the ciliary body. One of the most important objectives of the preoperative evaluation is to identify any underlying disorders responsible for postoperative glaucoma for which KPro is indicated. If these disorders are not identified and treated aggressively, visual improvements can be limited. Glaucoma shows an increased risk of postoperative vision loss following KPro surgery [4]. One study showed that 27 of the 36 eyes $(75 \%)$ that did not have glaucoma diagnosed before KPro surgery developed glaucoma de novo postoperatively [5]. Risk factors for the development of postoperative glaucoma include pre-existing glaucoma, the presence of PAS with or without other anterior segment abnormalities, crowding of the anterior chamber angle and chronic inflammation. The use of topical steroids is also associated with increased 
intraocular pressure, which occurs in $30 \%$ of the normal population [6]. The prevention of postKPro glaucoma begins by controlling any preexisting glaucoma preoperatively. Usually, this condition can be managed with glaucoma medications. In cases with recalcitrant glaucoma, coexistent corneal disease and glaucoma in ocular burns may be managed by either drainage surgery and subsequent implantation of KPro or a combined glaucoma drainage device at the time of KPro implantation. Aldave reported that a significantly greater percentage of eyes in the KPro alone group demonstrated worsening of the IOP 1 year after surgery compared with that in the KPro plus tube group [7]. Cortina reported that compared with KPro alone, KPro combined with tube surgery resulted in lower rates of additional surgical procedures and similar visual outcomes at 1 year [8].

This study aimed to report our experience with patients with ocular burns treated with implantation of KPro with the prophylactic pars plana Ahmed glaucoma valve (AGV; New World MedicalInc, Rancho Cucamonga, CA, USA) versus implantation with KPro alone. We were particularly interested in the development of new onset glaucoma postoperatively.

\section{METHODS}

The present study was approved by the Institutional Review Board of Zhongshan Ophthalmic Center and conformed to the tenets of the Declaration of Helsinki. A retrospective chart review was performed on all eyes that received KPro surgery at Zhongshan Ophthalmic Center between January 1, 2012, and December 30, 2019, using the Boston KPro type 1 threadless design with an $8.5 \mathrm{~mm}, 16$-hole backplate. Patients with a history of ocular burns were included in the analysis. Glaucoma surgery was performed in these eyes as concomitant or subsequent procedures.

The patients were divided into two groups based on the time of AGV surgery: Group KPro alone (Group 1) included patients with subsequent or no AGV surgery after KPro implantation; Group KPro combined with AGV (Group 2) included patients who had undergone concomitant pars plana AGV surgery at the time of KPro surgery. Patients with previously diagnosed glaucoma before KPro surgery were excluded from the present study. Preoperative glaucoma was defined as a history of a durable elevated IOP $\geq 25 \mathrm{mmHg}$ at different time points, which resulted in the introduction of anti-glaucoma medication or surgical intervention. Eyes in which the $C / D$ ratio could not be evaluated postoperatively were also excluded.

Preoperatively, patients had undergone ultrasound biomicroscopy (UBM) to identify PAS changes in the anterior chamber and ultrasonography to evaluate posterior segment pathology before KPro implantation. The preoperative patient demographics included age, sex, type of ocular burn injury, number of prior corneal transplants, best-corrected visual acuity (BCVA) and IOP. The data concerning polymethylmethacrylate (PMMA) or titanium backplate, intraoperative procedures, postoperative BCVA, $C / D$ ratio and use of antiglaucoma medication were also documented. The surgery was performed using the same standard technique in Group 1 [9]. In Group 2, concomitant with the KPro procedure, ten eyes had undergone simultaneous pars plana AGV implantation. The surgical techniques have been described previously [10]. Briefly, all the patients had undergone pars plana vitrectomy using a wide-field Landers temporary keratoprosthesis at the time of tube insertion. Care was taken to trim the vitreous base in the area of intended tube insertion. After KPro was implanted, a scleral fistula was performed $4.0-4.5 \mathrm{~mm}$ posterior to the limbus using a $23 \mathrm{G}$ needle, and the tube was introduced. The tube was covered with a patch graft of alcohol-preserved donor sclera or a corneal button from the patient. Since 2018, the authors have transitioned to coimplantation of an AGV at the time of KPro surgery in ocular burn eyes without preexisting glaucoma.

Glaucoma de novo was defined as progressive enlargement of the $C / D$ ratio $\geq 0.2$ from any prior postoperative visit [11]. IOP was measured preoperatively by Goldmann applanation tonometry or finger palpation and postoperatively by finger palpation and registered as the mean when a range was recorded. 
Table 1 Baseline characteristics of patients in the KPro group versus KPro + AGV group

\begin{tabular}{|c|c|c|c|}
\hline & KPro group & KPro + AGV group & $p$ \\
\hline Patients, $n$ & 16 & 10 & \\
\hline \multicolumn{4}{|l|}{ Baseline demographics } \\
\hline Age (years), mean $\pm S D$ & $40.2 \pm 10.6$ & $46.3 \pm 12.4$ & $0.19^{\mathrm{a}}$ \\
\hline Preoperative BCVA, mean (range) & $\mathrm{LP}(\mathrm{HM}$ to $\mathrm{LP})$ & $\mathrm{LP}(\mathrm{FC}$ to $\mathrm{LP})$ & \\
\hline Right eye & 9 & 5 & $0.75^{\mathrm{b}}$ \\
\hline \multicolumn{4}{|l|}{ Preoperative ocular status } \\
\hline Preoperative IOP $(\mathrm{mmHg} \pm \mathrm{SD})$ & $12.0 \pm 3.3$ & $12.9 \pm 3.1$ & $0.49^{\mathrm{a}}$ \\
\hline Mean clock of anterior synechie & $1.88 \pm 1.63$ & $2.30 \pm 1.83$ & $0.54^{\mathrm{c}}$ \\
\hline \multicolumn{4}{|l|}{ LSCD } \\
\hline Stage Ib & 9 & 5 & $0.75^{\mathrm{b}}$ \\
\hline Stage IIb & 7 & 5 & \\
\hline \multicolumn{4}{|l|}{ Lens status } \\
\hline Phakic & 12 & 7 & $0.56^{\mathrm{b}}$ \\
\hline Aphakic & 4 & 3 & \\
\hline \multicolumn{4}{|l|}{ Diagnostic category } \\
\hline Thermal burn & 4 & 3 & $0.82^{\mathrm{b}}$ \\
\hline Acid chemical burn & 5 & 2 & \\
\hline Alkali chemical burn & 7 & 5 & \\
\hline \multicolumn{4}{|l|}{ Ocular history } \\
\hline Prior intraocular surgeries & 10 & 5 & $0.53^{\mathrm{b}}$ \\
\hline Prior graft failure & 10 & 4 & $0.23^{\mathrm{b}}$ \\
\hline Prior corneal transplants, mean $\pm S D$ & $0.94 \pm 1.12$ & $0.50 \pm 0.70$ & $0.28^{\mathrm{c}}$ \\
\hline \multicolumn{4}{|l|}{ Backplate type } \\
\hline PMMA & 10 & 1 & $0.01^{\mathrm{b}}$ \\
\hline Titanium & 6 & 9 & \\
\hline \multicolumn{4}{|l|}{ Corneal carrier } \\
\hline AUTO & 3 & 0 & $0.21^{\mathrm{b}}$ \\
\hline ALLO & 13 & 10 & \\
\hline Time (KPro-ocular injury, y) & $4.88 \pm 2.94$ & $4.70 \pm 1.76$ & $0.86^{\mathrm{a}}$ \\
\hline
\end{tabular}

Bold value indicates $p<0.05$

$B C V A$ best corrected visual acuity; $C F$ counting fingers; $H M$ hand motion; $L P$ light perception; $S D$ standard deviation; $L S C D$ limbal stem cell deficiency; $A U T O$ autograft; $A L L O$ allograft

a Student's $t$-test

${ }^{\mathrm{b}}$ Fisher exact test

${ }^{\mathrm{c}}$ Mann-Whitney $U$ test 


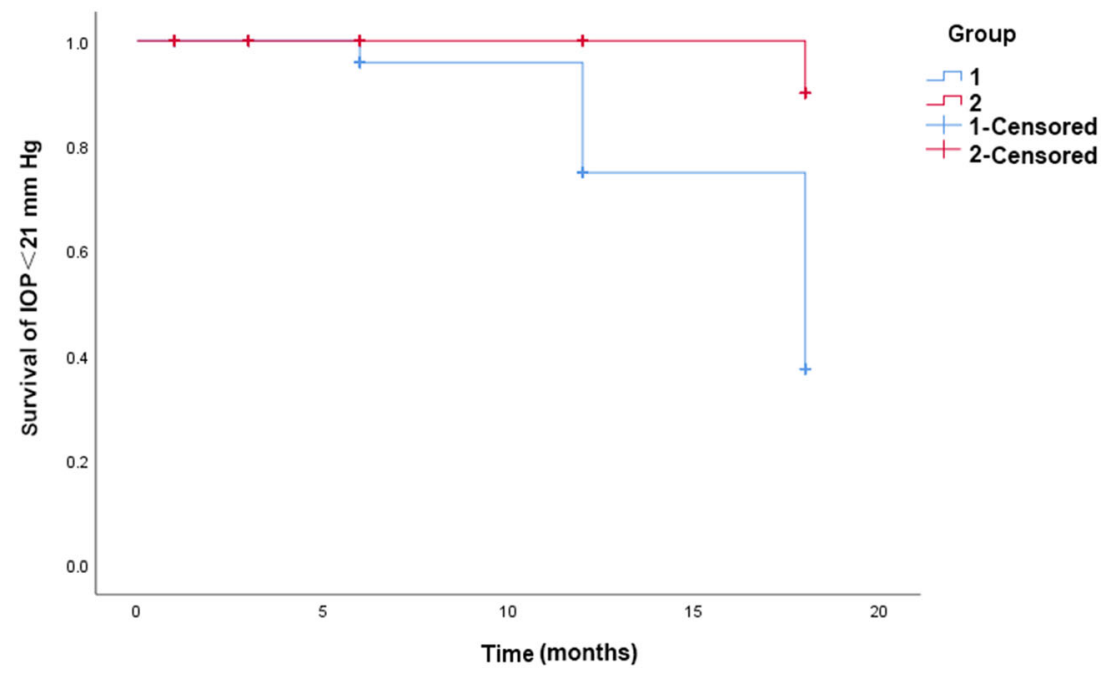

Fig. 1 Role of combined Ahmed glaucoma valve surgery in KPro-implanted eyes without glaucoma preoperatively. For the Kaplan-Meier estimate of the probability of maintaining IOP $<21 \mathrm{mmHg}$ across time in two groups, eyes with tube surgery (red line, Group 2) showed a significantly slower development of $\mathrm{IOP} \geq 21 \mathrm{mmHg}$ $(P<0.01$, log-rank test $)$
The postoperative $C / D$ ratio was recorded at the slit-lamp examination using a 90-diopter handheld lens. A small beam is adjusted to the vertical diameter of the optic disc, and its length is read on the scale of the slit-lamp. The optic cup was determined by its contour, with the outer margin taken to be the point where its wall met the plane of the disc surface. The direction or point of deviation of small blood vessels on the surface of the optic nerve head is used to determine the size of the optic cup. The vertical $C / D$ ratio was then calculated.

Postoperatively, all eyes received tobramycin dexamethasone eye drops, four times daily, for

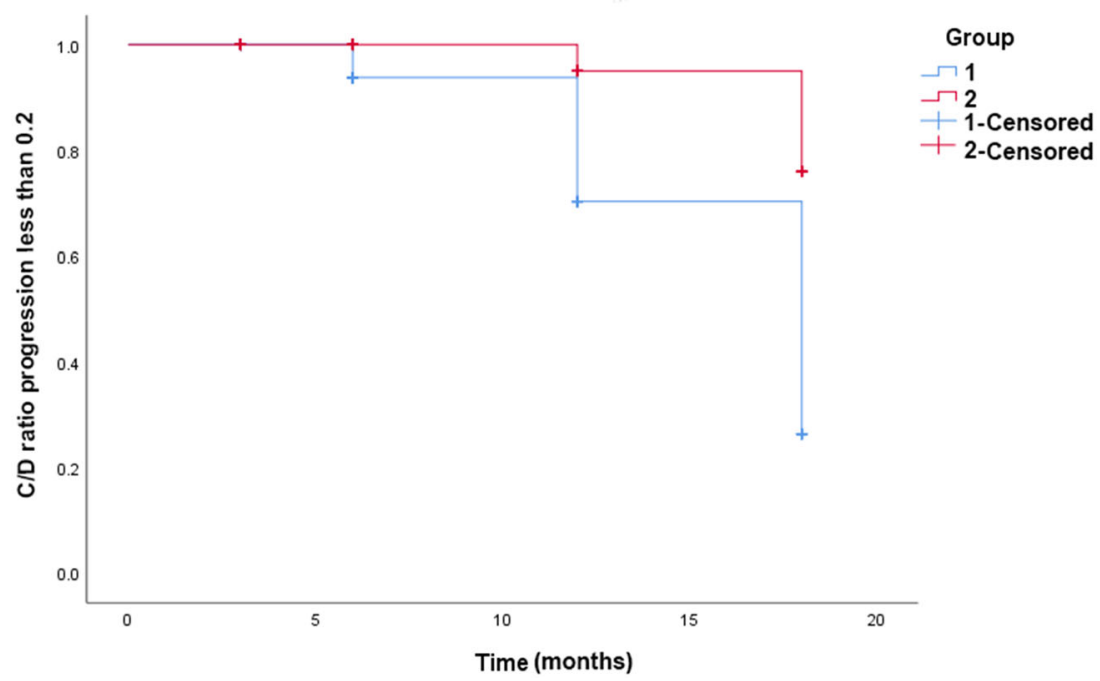

Fig. 2 Kaplan-Meier survival analysis showed $C / D$ ratio progression $<0.2$ for both groups. Eyes with tube surgery (red line, Group 2) had significantly higher rates of $C / D$ ratio progression $<0.2(P<0.01$, log-rank test $)$ 
the first month. Then, the topical steroid fluoroquinolone was tapered to twice daily every day over a 3-month period, and daily fluoroquinolone was used indefinitely for both groups. All the eyes were maintained on fourthgeneration fluoroquinolones indefinitely. Patients were followed up postoperatively at day 1 , weeks 1 and 2, months 1,3 and 6 , and every 3 months thereafter. To effectively compare glaucoma and other complications between the groups, all the patient data were compared for the same follow-up period. Eighteen months was chosen because this period was the minimal follow-up time for one patient in Group 2. Successful glaucoma control was defined as no further glaucoma surgery and an IOP between 5 and $21 \mathrm{mmHg}$ with or without glaucoma medications. Combination medication eye drops were counted as two medications in data analyses. Visual acuity was measured using a Snellen chart at every visit. The BCVA was converted to the $\log$ MAR scale for statistical analysis. Count fingers (CF), hand motion (HM) and no light perception (NLP) visual acuity were converted to $\log$ MAR 1.8, 2.3 and 2.6, respectively [12]. The primary outcome measure of this study was postoperative glaucoma at 18 months of followup. Secondary outcome measures include postoperative visual acuity and device retention.

Statistical analysis was performed using SPSS 25.0 for Windows (IBM SPSS Statistics for Windows, Version 25.0; IBM Corp, Armonk, NY, USA). Two-tailed $t$-test was used to compare normally distributed continuous variables. The Mann-Whitney $U$ test was performed to compare nonnormally distributed variables. Fisher's exact test was used to compare categorical variables. Kaplan-Meier survival curves were used for the $C / D$ ratio, and the log-rank test was used to compare the survival probability of $\mathrm{C} /$ $D$ ratio progression $<0.2$ for both groups. Statistical significance was defined at $P<0.05$.

\section{RESULTS}

A total of 26 eyes of 26 patients, all of whom were male, were included in this study. The patient demographics and initial ocular characteristics are summarized in Table 1 . The patients ranged in age from 27 to 67 years (mean, $42.7 \pm 11.3$ years), with no difference between the groups. All the patients were injured by ocular chemical or thermal burns. The mean time between KPro implantation and ocular injury was $4.8 \pm 2.5$ years. No significant difference was found in the time between KPro implantation and ocular injury in the two groups $(P=0.86)$. The staging of severe ocular surface disease based on limbal stem cell deficiency (LSCD) was comparable in both groups. The two groups were similar in the proportions of ocular burn type or intraocular surgeries before KPro implantation. Ten patients in Group 1 and four patients in Group 2 had undergone prior corneal transplants $(P=0.38)$. All the patients showed a stable IOP (range, 7-19 $\mathrm{mmHg}$ ) and no obvious optic disc cupping by $\mathrm{B}$ ultrasound before KPro implantation. A higher proportion of eyes with PMMA backplates was found in the KPro alone group than in the KPro combined with AGV group (10 of 16 vs. 1 of 10 eyes, respectively; $P=0.01$ ).

\section{Glaucoma Outcome}

All the implanted glaucoma drainage devices were AGVs because of the limited subconjunctival space in our ocular burn patients. The mean pre-KPro IOP was $12.0 \pm 3.3 \mathrm{mmHg}$ in Group 1. At the 18-month follow-up, the mean IOP was $20.5 \pm 4.8 \mathrm{mmHg}$ on a mean of $2.1 \pm 1.0$ glaucoma medications. Of the 8 eyes that developed an IOP $>21 \mathrm{mmHg}$ postoperatively in Group 1, five eyes received transscleral diode cyclophotocoagulation, and two were treated with AGV implantation. One patient rejected undergoing glaucoma surgery and was maintained on topical antiglaucoma eye drops. At the 18-month follow-up of the eight eyes, the mean IOP was $24.6 \pm 1.7 \mathrm{mmHg}$ on a mean of $3.0 \pm 0.0$ glaucoma medications. The IOP was controlled in $87.5 \%, 56.2 \%$ and $50.0 \%$ of eyes at 6,12 and 18 months, respectively, in Group 1. The mean pre-KPro IOP was $12.9 \pm 3.1 \mathrm{mmHg}$ in Group 2. One eye developed an IOP $>21 \mathrm{mmHg}$ in Group 2 postoperatively because of conjunctival scarring around the valve. At the 18-month follow-up, the mean 
IOP was $17.3 \pm 5.6 \mathrm{mmHg}$ on a mean of $1.3 \pm 0.8$ glaucoma medications. AGV implantation successfully controlled the IOP in $100 \%$, $100 \%$ and $90.1 \%$ of eyes at 6,12 and 18 months, respectively. The overall survival of patients with an IOP $<21 \mathrm{mmHg}$ was analyzed using a Kaplan-Meier curve (Fig. $1 ; P<0.01$; log-rank test). At the 18-month follow-up, more eyes in Group 1 had an IOP $>21 \mathrm{mmHg}(8 / 16)$ than in Group $2(1 / 10 ; P=0.04)$, and the proportion of eyes treated with glaucoma surgery after KPro implantation was higher $(7 / 16$ vs. $0 / 10$; $P=0.02$ ).

The baseline $C / D$ ratio was assessed within 1 month postoperatively because of poor visualization preoperatively. The baseline mean $C /$ $D$ ratio was 0.37 (range, $0.30-0.50 ; \mathrm{SD}, 0.06$ ) in Group 1 and 0.40 (range, 0.30-0.80; SD, 0.15) in Group $2(P=0.45)$. At the final follow-up, more eyes in Group 1 had eyes with a $C / D$ ratio $\geq 0.8$ $(7 / 16)$ than in Group $2(2 / 10, \quad P=0.21)$, although the difference was not statistically significant. A $C / D$ ratio progression of $\geq 0.2$ was observed in $62.5 \%(10 / 16)$ of Group 1 and $20 \%(2 / 10)$ of Group $2(P=0.04)$. The KaplanMeier survival analysis showed that the $C /$
$D$ ratio progression was $<0.2$ for both groups (Fig. 2; $P<0.01$, log-rank test).

\section{Visual Outcome}

Preoperative vision was counting fingers or worse in all eyes. The mean BCVA postoperatively in Group 1 was 0.59 at 3 months (range, $0.1-1.0$ ), 0.91 at 6 months (range, $0.1-1.8$ ) and 1.20 at 12 months (range, $0.3-1.8$ ). The BCVA at 18 months for Group 1 was 1.32 (range, 0.3-2.8). Comparing preoperative BCVA with BCVA at 18 months in Group 1, vision improved in 13 of 16 eyes and was unchanged in 1 eye. Vision worsened in two eyes, which became NLP because of glaucoma. The mean BCVA postoperatively in Group 2 was 0.93 at 3 months (range, 0.3-2.3), 0.93 at 6 months (range, 0.3-2.3) and 0.85 at 12 months (range, $0.2-2.3$ ). The BCVA at 18 months for Group 2 was 1.03 (range, 0.3-2.3). Comparing preoperative BCVA with BCVA at 18 months in Group 2 , vision improved in eight of ten eyes and was unchanged in one eye. Vision worsened in one eye. No eye became NLP because of glaucoma in Group 2. Figure 3 shows the analysis of VA

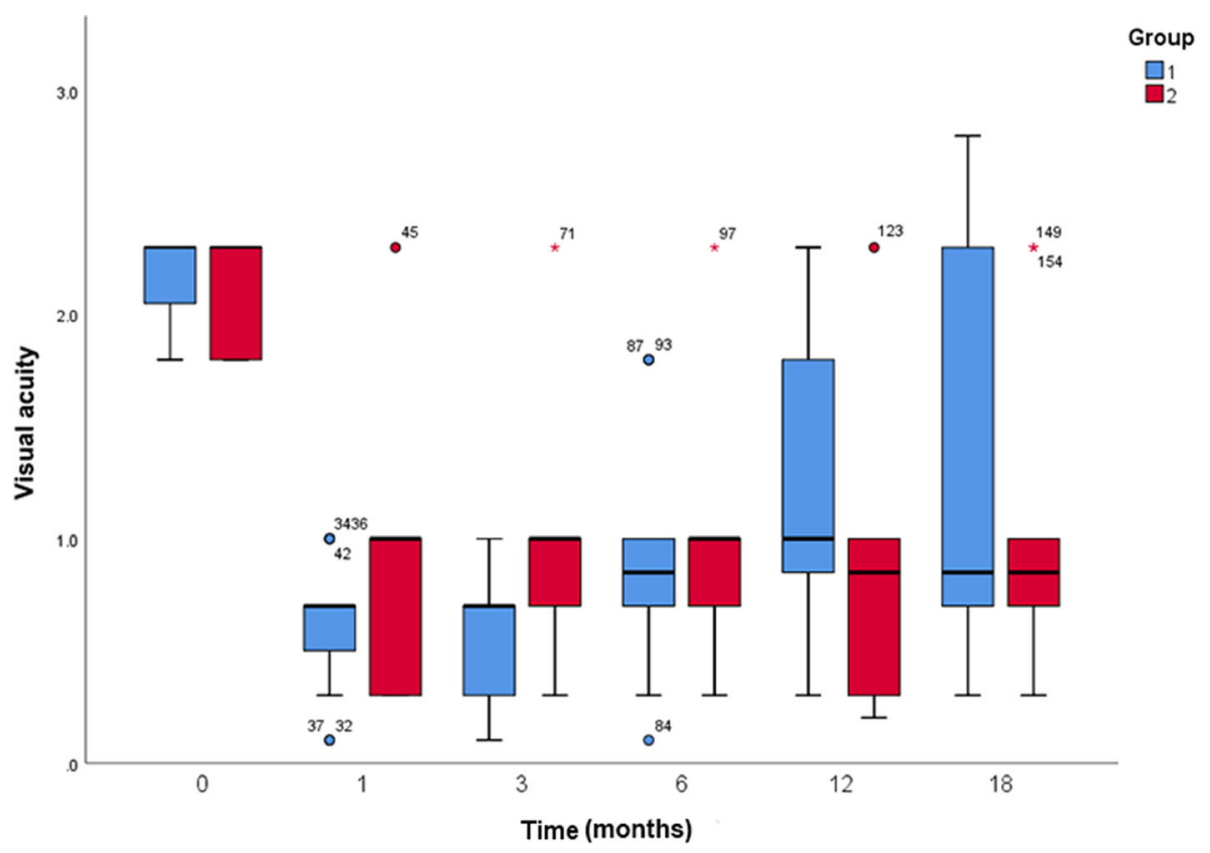

Fig. 3 Box plot of visual acuity in patients with KPro alone (blue, Group 1) versus KPro combined AGV (red, Group 2) over the 18-month follow-up period 
Table 2 Complication rates for group 1 versus group 2

\begin{tabular}{llll}
\hline $\begin{array}{l}\text { Postoperative } \\
\text { complication }\end{array}$ & $\begin{array}{l}\text { Group 1 } \\
n=16 \\
\text { eyes }\end{array}$ & $\begin{array}{l}\text { Group 2 } \\
\boldsymbol{n}=\mathbf{1 0} \\
\text { eyes }\end{array}$ & $\boldsymbol{p}$ \\
\hline $\begin{array}{l}\text { Retroprosthetic } \\
\text { membrane }\end{array}$ & $6(37.5 \%)$ & $2(20 \%)$ & 0.31 \\
$\begin{array}{l}\text { Sterile corneal melt } \\
\text { Cystoid macular edema }\end{array}$ & $1(6.3 \%)$ & $1(10 \%)$ & 0.63 \\
Sterile vitritis & $0(0 \%)$ & $1(10 \%)$ & 0.38 \\
Vitreous hemorrhage & $1(6.3 \%)$ & $1(10 \%)$ & 0.63 \\
Tube obstruction & $1(6.3 \%)$ & $0(0 \%)$ & 0.59 \\
Glaucoma de novo & 10 & $2(20 \%)$ & $\mathbf{0 . 0 4}$ \\
& $(62.5 \%)$ & & 0.38 \\
\hline
\end{tabular}

Bold value indicates $P<0.05$

outcomes based on the two groups; no significant difference was found in VA between the groups at postoperative follow-up times of 3,6 , 12 and 18 months.

\section{Complications}

After KPro implantation, $68.7 \%$ of eyes in Group 1 and $50.0 \%$ of eyes in Group 2 developed one or more complications. The most frequent postoperative complication besides glaucoma was retroprosthetic membrane (RPM) formation. RPM formation was observed in six (37.5\%) eyes in Group 1(Table 2). Three of those eyes were treated successfully with Nd:YAG laser membranotomy. The visual acuity of the other eyes did not change much; only observation was needed. Other complications in Group 1 were corneal sterile melting in one (6.3\%) eye and vitreous hemorrhage in one $(6.3 \%)$ eye. In Group 2, complications occurred at the following frequencies: RPM in two(20.0\%) eyes, sterile vitritis in one(10.0\%) eye (Fig. 4), vitreous hemorrhage in one $(10.0 \%)$ eye, cystoid macular edema in one $(10.0 \%)$ eye and corneal sterile melting in one (10.0\%) eye. An additional concern with a high IOP after KPro is the mechanical stress placed on the wound, which can contribute to wound ectasia. One patient in
Group 1 developed de novo glaucoma and a bulge of the cornea with a risk of corneal erosion 10 months after KPro surgery (Fig. 5).This patient had undergone pars plana AGV implantation through the $3.5-\mathrm{mm}$ optical surface of KPro. Postoperatively, with a reduced IOP, the protrusion of the cornea was alleviated, and no gap developed between KPro and the corneal carriers.

\section{DISCUSSION}

Ocular burns are a critical problem because they may destroy the entire corneal epithelium and extend into the fornices. Eyes injured by burns may develop various ocular diseases, including disabling corneal opacities, severe scarring with neovascularization, intraocular inflammation and iris ischemia. The most critical avoidable complication is secondary glaucoma, which occurs in $22 \%$ of eyes with severe ocular chemical burns [13]. Patients with ocular burns are at risk of developing glaucoma because of heterogeneous mechanisms, including structural damage to the trabecular meshwork, robust and persistent ocular inflammation, corticosteroid response, secondary opacity of the lens and peripheral anterior synechiae with or without papillary block. Glaucoma recalcitrant to medical therapy may also develop in the late phase of ocular burns. Trabeculectomy has difficulty allowing bleb survival because of extensive scarring of the perilimbal and bulbar conjunctiva. The results of cyclophotocoagulation of the ciliary body are unpredictable and may be associated with side effects of inflammation, choroidal effusions, hypotony and even phthisis. The AGV has been demonstrated to be a safe and efficacious device in patients with refractory glaucoma and an increased risk of surgical failure. It is efficient at lowering the IOP and reducing the number of medications [14].

In recent years, KPro has been used successfully as surgeons have observed improved outcomes under conditions in which standard penetrating keratoplasty has a poor prognosis, such as ocular burns. The advantages of KPro include the elimination of endothelial cell 

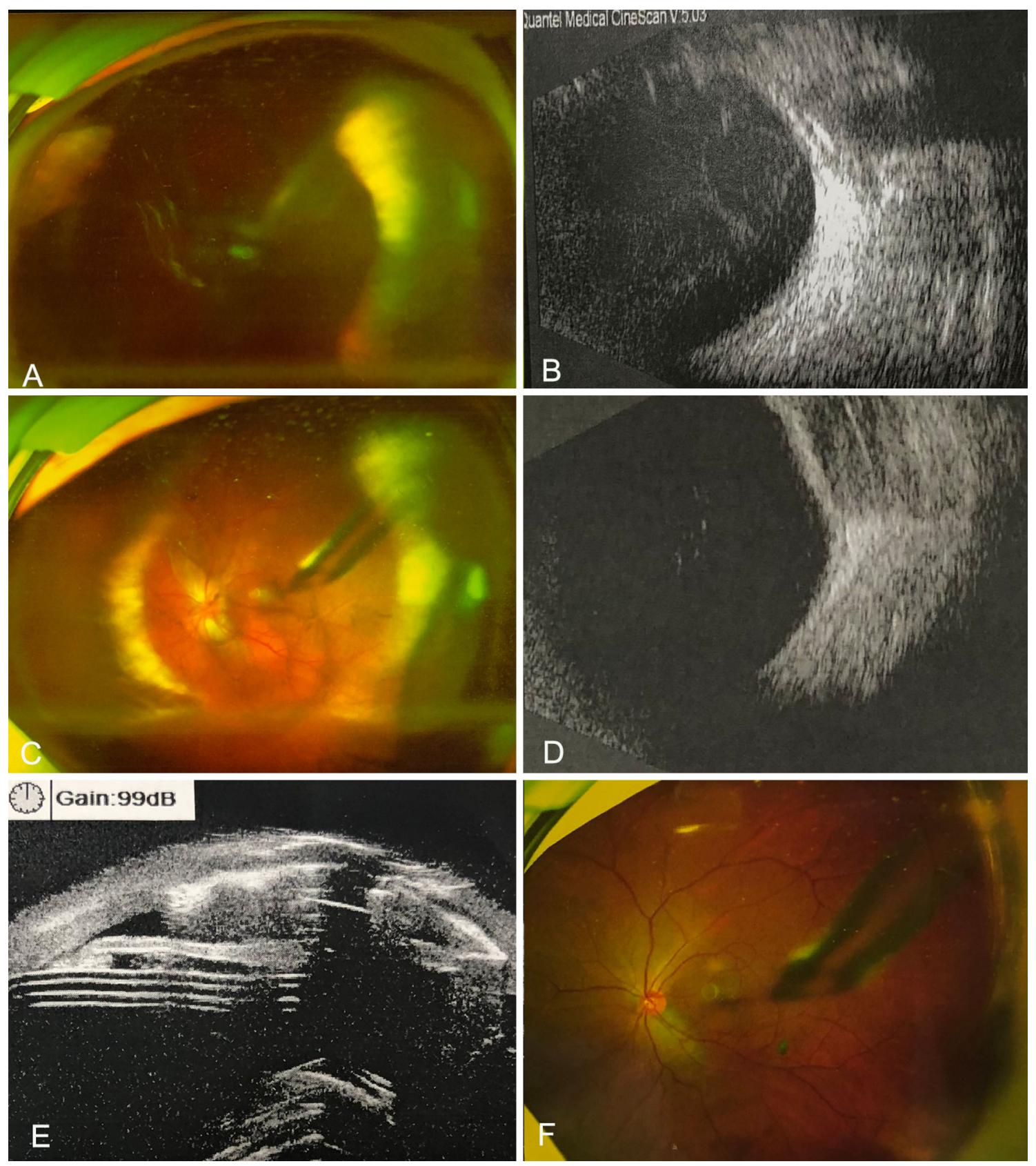

Fig. 4 Sterile vitritis after KPro combined AGV surgery. A Sterile vitritis occurred 2 months postoperatively, Optomap $^{\circledR}$ image of the patient showing a hazy media and tube superotemporally. B B-scan ultrasound showing the vitreous cavity was filled with increased amplitude point echoes. C, D After 2-week treatment of systemic prednisone and topical tobramycin dexamethasone,

Optomap ${ }^{\circledR}$ image of the patient shows the hazy media reduced, and B-scan ultrasound shows echolucent fluid areas around the plate and minimal echogenicity in the vitreous cavity suggestive of decreasing vitritis. E Ultrasound biomicroscopy showing contact of the tube with the ciliary body. F Two months after treatment, the vitritis resolved and the media cleared 

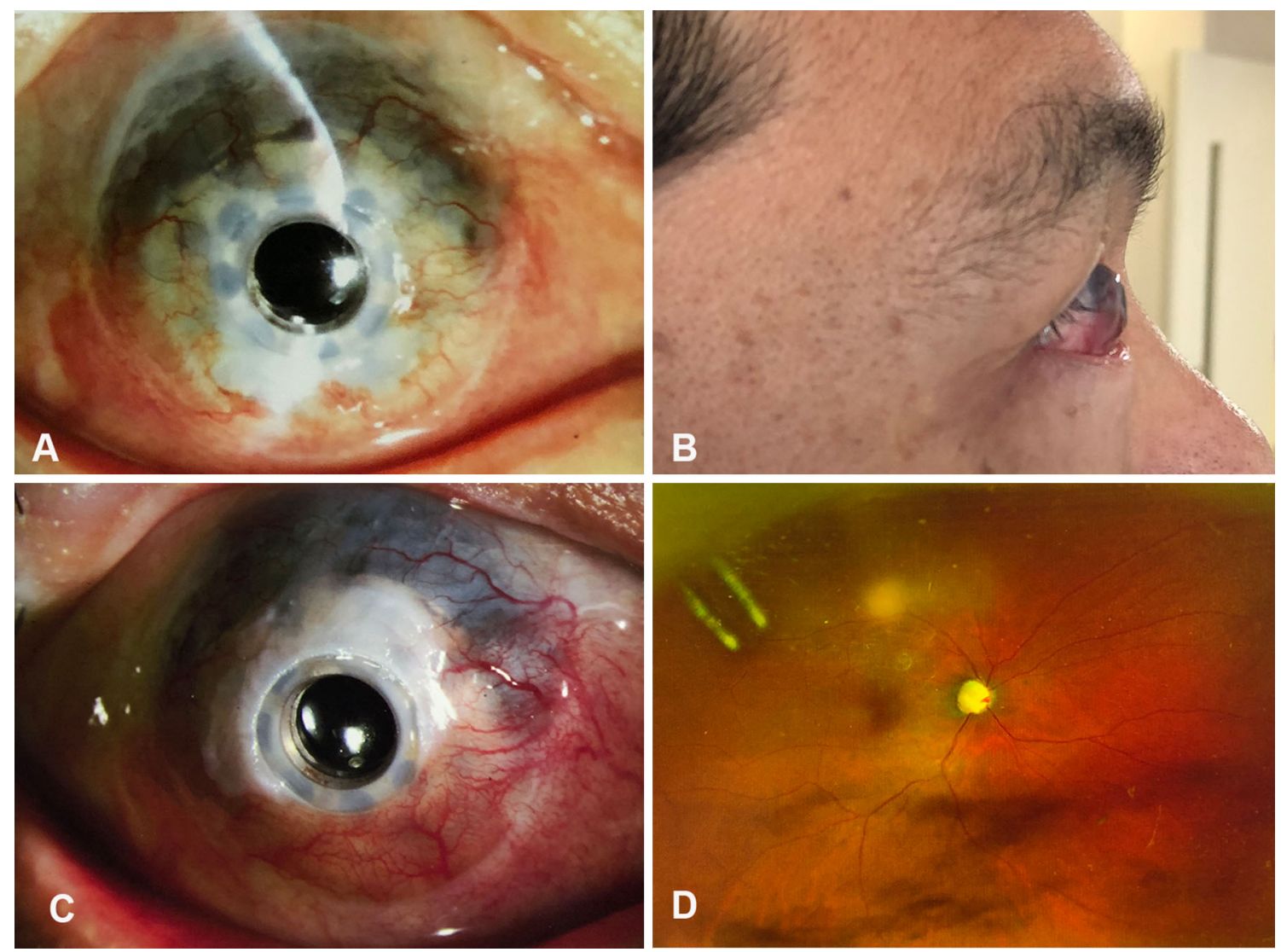

Fig. 5 Patient developed glaucoma de novo in group KPro alone. A, B Eyes developed high IOP and protrusion of the cornea 10 months after KPro implantation. C One year after pars plana AGV surgery, with the drop of IOP and stabilization of protrusion of the cornea, the edge of the

front plate was covered with corneal epithelium. D Optomap $^{\circledR}$ image of the patient showing the tube superotemporally and pale disc with nasal hemorrhage postoperatively

rejection and decreased dependence on the limbal stem cell status. Patients who cannot maintain systemic immunosuppression drugs may also be candidates for this device. The success in improvement outcomes using the KPro can be attributed to device design modification and advances in postoperative management. However, despite these innovations, KPro carries continued risks of glaucoma, compromising visual rehabilitation after an otherwise successful KPro procedure. Regardless of whether ocular burns have a strong association with glaucomatous disease, the KPro backplate may also cause angle crowding with progressive peripheral anterior synechiae and subsequent angle closure in nonglaucomatous individuals [15]. Additionally, the optic and backplate of

KPro enhance scleral rigidity, which may result in glaucoma progression, despite a normal IOP [16]. The KPro devices used in the present study were the aphakic type, which demands anterior vitrectomy to be performed in Group 1. Vitrectomy was hypothesized to cause late-onset open-angle glaucoma from increased oxidative damage to the trabecular meshwork, particularly in aphakic eyes $[17,18]$. Additionally, the elevation of inflammatory cytokines in KPro eyes may result in retinal ganglion cell death [19]. The manufacturer of KPro has recommended aggressive control of glaucoma to maintain a target IOP $\leq 12 \mathrm{mmHg}$ [7].

In our study, placement of a glaucoma drainage device using the pars plana approach combined with KPro implantation in ocular 
burns without preexisting glaucoma resulted in decreased new onset of glaucoma, which is an essential cause of visual decline after KPro surgery. KPro combined with AGV implantation also effectively decreased secondary glaucoma surgery compared with KPro alone. Although the surgical time of combined AGV and KPro surgeries was longer than that of KPro-alone surgeries, the intra- and postoperative complications were comparable in the two groups. We prefer to insert the AGV tube while vitrectomy infusion is performed. A better view of the tube is obtained in the vitreous cavity, and a substantial drop in the IOP is prevented. Prolonged hypotony during surgery may result in anterior synechiae, which is a risk factor for secondary glaucoma [20]. Placement of a tube using par plana has several advantages, as described by previous authors [8]: (1) adequate contact lens fitting and elimination of friction between the contact lens and AGV plate/tube; (2) decreased risk of tube obstruction in the limited space in the anterior chamber after KPro implantation. We also noticed that with the development of wide-field retinal photographs, the opening of the tube could be visualized directly. If the tube was placed in the anterior chamber, it had to be placed posterior to the optical stem to visualize the opening of the tube. This set-up will interfere with the vision of the patient. If the tube opening was out of the axis of the PMMA stem, it was difficult to evaluate the tube using anterior segment optical coherence tomography or UBM.

In the combined surgery, wide-field temporary keratoprosthesis offers a better view to shave the vitreous base $360^{\circ}$ before implantation of the AGV and KPro. Glaucoma occurring after KPro surgery is more challenging for surgical intervention. Pars plana vitrectomy may be performed using a $3.5-\mathrm{mm}$ optical stem of the KPro, with scleral depression for optimal visualization of the vitreous base. In one patient in Group 1, de novo glaucoma with a high IOP caused severe visual loss and corneal carrier protrusion, endangering KPro retention. Although pars plana AGV surgery after KPro implantation can only keep the VA of this patient at light perception, it effectively lowered the IOP and alleviated the bulge of the corneal carrier.

Sterile vitritis occurred in one patient after combined KPro and AGV implantation 2 months postoperatively. He was treated with topical prednisolone acetate 1\% eyedrops and systemic prednisone, and chronic vitreous inflammation resolved within 2 months. Sterile vitritis is a poorly understood complication with an undefined etiology. It may be caused by periprosthetic tissue gaps [21], micromotion of the KPro within the cornea, viral uveitis or noninfectious inflammation triggered by the microbial cell wall. The first series of sterile vitritis was reported by Nouri, in which five patients had undergone combined AGV and KPro surgery [22]. Our patient was implanted with an FP8-type AGV with no fenestration in the plate because of the restricted space of the conjunctiva. Postoperatively, UBM examination revealed contact of the tube with the ciliary body. We presumed that tube-ciliary body chafing might irritate the uveal tissue, leading to a breakdown of the blood-aqueous barrier, releasing cytokines to trigger an inflammatory cascade causing sterile vitritis [23].

The limitation of this study is the retrospective nature of the data collection, short followup, small sample size and bias that may exist due to patient selection. Because of the difference in the availability of KPro with PMMA and titanium backplate, more eyes in Group 2 were implanted with KPro with titanium backplate than eyes in Group 1. However, one study found that different materials of the KPro backplate did not have a significant impact on the angle anatomy [24].

\section{CONCLUSIONS}

In summary, KPro surgery is an effective option to rehabilitate vision in eyes with ocular burns, given the high risk of new-onset glaucoma after KPro implantation alone. KPro combined with 
pars plana AGV implantation may help to lower glaucoma de novo in patients injured with ocular burns.

\section{ACKNOWLEDGEMENTS}

We hereby thank all the participants of the study.

Funding. No funding or sponsorship was received for this study or publication of this article.

Medical Writing Assistance. Language editing and assistance for this article were provided by AJE and funded by the authors.

Authorship. All named authors meet the International Committee of Medical Journal Editors (ICMJE) criteria for authorship for this article, take responsibility for the integrity of the work as a whole, and have given their approval for this version to be published.

Author Contributions. Conceptualization, Jianjun $\mathrm{Gu}$ and Jiaqi Chen; Jiaqi Chen, Jianjun $\mathrm{Gu}$, Ting Huang, Yuying Zhang, Lixia Lin, Zhancong Ou, Miao Chen, Jin Zhou, Liangbo Zeng, and Yuwei $\mathrm{Xu}$ are responsible for the treatment and follow-up; Lixia Lin, Zhancong Ou, Miao Chen, Jin Zhou, Liangbo Zeng, and Yuwei $\mathrm{Xu}$ are responsible for the interpretation of data; Jianjun $\mathrm{Gu}$ and Jiajie Zhai wrote the main manuscript text; resources, Jiajie Zhai and Jiaqi Chen. All authors have read and agreed to the published version of the manuscript.

Disclosures. Jianjun Gu, Yuying Zhang, Jiajie Zhai, Lixia Lin, Zhancong Ou, Ting Huang, Miao Chen, Jin Zhou, Liangbo Zeng, Yuwei Xu, Jiaqi Chen all confirm that they have no conflicts of interest to disclose.

Compliance with Ethics Guidelines. This study was conducted in accordance with the 1964 Declaration of Helsinki and its later amendments and was approved by the Ethics Committee of Zhongshan Ophthalmic Center (2019QXPJ004). Informed consent for participation and publication of patient data was obtained from all individual participants included in the study. Permissions have been obtained from our patients to publish the images of patients in this article.

Data Availability. The datasets generated during and/or analyzed during the current study are available from the corresponding author on reasonable request.

Open Access. This article is licensed under a Creative Commons Attribution-NonCommercial 4.0 International License, which permits any non-commercial use, sharing, adaptation, distribution and reproduction in any medium or format, as long as you give appropriate credit to the original author(s) and the source, provide a link to the Creative Commons licence, and indicate if changes were made. The images or other third party material in this article are included in the article's Creative Commons licence, unless indicated otherwise in a credit line to the material. If material is not included in the article's Creative Commons licence and your intended use is not permitted by statutory regulation or exceeds the permitted use, you will need to obtain permission directly from the copyright holder. To view a copy of this licence, visit http://creativecommons.org/licenses/by$\mathrm{nc} / 4.0 /$.

\section{REFERENCES}

1. Harissi-Dagher $\mathrm{M}$, Dohlman $\mathrm{CH}$. The Boston keratoprosthesis in severe ocular trauma. Can J Ophthalmol. 2008;43(2):165-9. https://doi.org/10. 3129/i08-009.

2. Nascimento VD, De La Paz MF, Rosandic J, Stoiber J, Seyeddain O, Grabner G, De Toledo JA, Barraquer RI, Michael R. Influence of primary diagnosis and complications on visual outcome in patients receiving a Boston type 1 keratoprosthesis. Ophthalmic Res. 2014;52(1):9-16. https://doi.org/10. $1159 / 000358332$.

3. Kang JJ, de la Cruz J, Cortina MS. Visual outcomes of Boston keratoprosthesis implantation as the primary penetrating corneal procedure. Cornea. 
2012;31(12):1436-40. https://doi.org/10.1097/ICO. Ob013e31823f7765.

4. Ahmad S, Akpek EK, Gehlbach PL, Dunlap K, Ramulu PY. Predictors of visual outcomes following Boston type 1 keratoprosthesis implantation. Am J Ophthalmol. 2015;159(4):739-47. https://doi.org/ 10.1016/j.ajo.2014.12.024 (Epub 2014 Dec 30).

5. Crnej A, Paschalis EI, Salvador-Culla B, Tauber A, Drnovsek-Olup B, Shen LQ, Dohlman CH. Glaucoma progression and role of glaucoma surgery in patients with Boston keratoprosthesis. Cornea. 2014;33(4):349-54. https://doi.org/10.1097/ICO. 0000000000000067 .

6. Motolko MA. Phelps CD (1987) The secondary galucomas. Chapter 54. In: Duane TD, Jaeger EA, editors. Clinical ophthalmology. 3rd ed. Philadelphia: Harper and Row; 1987.

7. Lenis TL, Chiu SY, Law SK, Yu F, Aldave AJ. Safety of concurrent Boston Type I keratoprosthesis and glaucoma drainage device implantation. Ophthalmology. 2017;124(1):12-9. https://doi.org/10.1016/ j.ophtha.2016.08.003.

8. Jennifer ILLM, Andrea A, Faris IK, Robert H, Dingcai C, Marcia N, Thasarat SV, Soledad MC. Comparison of visual and anatomical outcomes of eyes undergonging type 1 Boston keratoprosthesis with combination pars plana vitrectomy with eyes without combination vitrectomy. Retina. 2018;38(Suppl 1): S125-33. https://doi.org/10.1097/IAE. 0000000000002036 .

9. Gu JJ, Zhai JJ, Zhou S, Chen J. Boston keratoprosthesis outcomes in severe ocular chemical burns in southern China: a retrospective study. Adv Ther. 2016;33(5):760-73. s12325-016-0330-9.

10. Huh ES, Aref AA, Vajaranant TS, de la Cruz J, Chau FY, Cortina MS. Outcomes of pars plana glaucoma drainage implant in Boston type 1 keratoprosthesis surgery. J Glaucoma. 2014;23(1):e39-44. https:// doi.org/10.1097/IJG.0b013e31829e55f8.

11. Nascimento E, Silva R, Shen LQ, Chiou CA, Shanbhag SS, Paschalis EI, Pasquale LR, Colby KA, Dohlman CH, Chodosh J, Alves MR. Glaucoma management in patients with aniridia and boston type 1 keratoprosthesis. Am J Ophthalmol. 2019;207:258-67. https://doi.org/10.1016/j.ajo. 2019.06.018.

12. Schulze-Bonsel K, Feltgen N, Burau H, Hansen L, Bach M. Visual acuities "hand motion" and "counting fingers" can be quantified with the freiburg visual acuity test. Invest Ophthalmol Vis Sci. 2006;47(3):1236-40. https://doi.org/10.1167/iovs.05-0981.
13. Tsai JH, Derby E, Holland EJ, Khatana AK. Incidence and prevalence of glaucoma in severe ocular surface disease. Cornea. 2006;25(5):530-2. https://doi.org/ 10.1097/01.ico.0000220776.93852.d9.

14. Torsten S, Focke Z, Karl UB-S. Pars plana-modified Ahmed Glaucoma Valve for treatment of refractory glaucoma: a pilot study. Graefes Arch Clin Exp Ophthalmol. 2006;244(3):336-41. https://doi.org/ 10.1007/s00417-005-0008-5.

15. Julian PSG Jr, de la Jose C, Richard BR, Douglas FB. Imaging implanted keratoprostheses with anteriorsegment optical coherence tomography and ultrasound biomicroscopy. Cornea. 2008;27(2):180-8. https://doi.org/10.1097/ICO.0b013e318159bc7d.

16. Julia CT, Younes A, Sébastien G, Krystel M, Mona H-D. Prevalence, progression, and impact of glaucoma on vision after Boston type 1 keratoprosthesis surgery. Am J Ophthalmol. 2012;153(2):267-274.e1. https://doi.org/10.1016/j.ajo.2011.07.022.

17. Larry K, Norihiko Y, Paulo E, Leslie MN, Irina VK, David CM, Stanley C. Incidence of, risk factors for, and combined mechanism of late-onset open-angle glaucoma after vitrectomy. Retina. 2012;32(1):160-7. https://doi.org/10.1097/IAE.0b013e318217fffb.

18. Chang S. LXII Edward Jackson lecture: open angle glaucoma after vitrectomy. Am J Ophthalmol. 2006;141(6):1033-43. https://doi.org/10.1016/j.ajo. 2006.02.014.

19. Eleftherios IP, Elise VT, James C, Louis RP, Kathryn C, Claes HD, Lucy QS. Blood levels of tumor necrosis factor alpha and its type 2 receptor are elevated in patients withboston type 1 keratoprosthesis. Curr Eye Res. 2019;44(6):599-606. https:// doi.org/10.1080/02713683.2019.1568500.

20. Onur K, Suleyman K, Gurkan E, Baran K, Sulay EO, Omer KD. Incidence of and risk factors for increased intraocular pressure after penetrating keratoplasty. Cornea. 2010;29(3):278-82. https://doi.org/10. 1097/ICO.0b013e3181b6eb9e.

21. Christina MG, Andrea C, Elise VT, Alja C, Kathryn AC, Claes HD, James C. Periprosthetic tissue loss in patients with idiopathic vitreous inflammation after the Boston keratoprosthesis. Cornea. 2015;34(11):1378-82. https://doi.org/10.1097/ICO. 0000000000000557.

22. Mahnaz N, Marlene LD, Claes HD. Sudden reversible vitritis after keratoprosthesis: an immune phenomenon? Cornea. 2005;24(8):915-9. https:// doi.org/10.1097/01.ico.0000159736.60424.ff.

23. Pinar CO, Magdi M, Jean D. Ultrasound biomicroscopy of pseudophakic eyes with chronic postoperative inflammation. J Cataract Refract Surg. 
2003;29(6):1185-91.

https://doi.org/10.1016/ s0886-3350(02)01920-X.

24. Taniguchi EV, Paschalis EI, Crnej A, Ren A, Colby KA, Chodosh J, Pasquale LR, Shen LQ, Dohlman
$\mathrm{CH}$, Cruzat A. The role of the back plate in angle anatomy with the Boston Type I keratoprosthesis. Cornea. 2017;36(9):1096-101. https://doi.org/10. 1097/ICO.0000000000001248. 